Meta

Journal des tradlucteurs

Translators' Journal

\title{
Problèmes culturels de la traduction d'Alicein Wonderland en français
}

\section{Claude Romney}

Volume 29, numéro 3, septembre 1984

URI : https://id.erudit.org/iderudit/003976ar

DOI : https://doi.org/10.7202/003976ar

Aller au sommaire du numéro

Éditeur(s)

Les Presses de l'Université de Montréal

ISSN

0026-0452 (imprimé)

1492-1421 (numérique)

Découvrir la revue

Citer cet article

Romney, C. (1984). Problèmes culturels de la traduction d'Alicein Wonderland

en français. Meta, 29(3), 267-280. https://doi.org/10.7202/003976ar d'utilisation que vous pouvez consulter en ligne.

https://apropos.erudit.org/fr/usagers/politique-dutilisation/ 


\section{PROBLÈMES CULTURELS DE LA TRADUCTION \\ D'ALICE IN WONDERLAND EN FRANÇAIS}

Claude RoMNEY

Il existe en français une quarantaine de versions différentes, aussi bien traductions qu'adaptations, du livre de Lewis Carroll Alice in Wonderland. Pourtant, de l'aveu des spécialistes, tant critiques qu'éducateurs, bibliothécaires et éditeurs, l'ouvrage n'a qu'un succès très relatif auprès du public français. Selon l'éminent angliciste Sylvère Monod, par exemple, "Alice est un ouvrage spécifiquement britannique qui déconcerte souvent le lecteur français et qui ne peut être pleinement goûté que de l'intérieur d'une conscience britannique ou britannisée ${ }^{1}$. 》

Cette incompréhension, cette déroute que suscite le livre vient en partie du fonds culturel qui sert de trame aux aventures de la fillette tombée dans le terrier du Lapin blanc. Souvent, le lecteur français ne saisit pas les allusions et se trouve plongé dans une atmosphère dont les éléments ne se rattachent pas à son monde à lui. Ce dépaysement est certainement plus gênant pour les enfants que pour les adultes qui acceptent plus volontiers l'existence de différences nationales. De fait, dans bien des cas, le traducteur doit décider s'il faut laisser telles quelles les références aux divers aspects culturels qu'il rencontre ou les transposer en les acclimatant. Son choix dépendra en grande mesure du public auquel il destine sa traduction : si elle s'adresse à des enfants, il est probable qu'elle contiendra davantage d'adaptations culturelles. À l'extrême, le caractère national de l'ouvrage se trouve oblitéré. Le plus souvent, cependant, les traducteurs ont procédé un peu au hasard, naturalisant tel élément, laissant subsister tel autre, ce qui produit un mélange hétéroclite qui désoriente tout autant le lecteur ${ }^{2}$. Nous avons examiné les problèmes culturels de la traduction d'Alice en français dans sept versions parues entre 1869 et $1976^{3}$ et avons constaté qu'elles adoptent des solutions variées.

1. Sylvère Monod (1970) : Histoire de la littérature anglaise de Victoria à Elizabeth II, Paris, Colin, p. 159.

2. Une thèse soutenue à Rennes en $\mathbf{1 9 7 8}$ a examiné des problèmes culturels, sans toutefois souligner la différence d'impressions produites par les traductions et l'original sur leurs lecteurs respectifs. Il s'agit de la thèse d'Alain Chapel, Équivoque et traduction dans "Alice au pays des merveilles ", Université de Haute-Bretagne-Rennes II, 1978.

3. Les versions françaises que nous avons étudiées sont les suivantes :

- Traduction d'Henri Bué (1972) : New York, Dover Publications. (Fac-similé de la 1 éd., Londres, Macmillan, 1869.)

- Traduction de Marie-Madeleine Fayet (1930) : Paris, Les CEuvres représentatives.

- Traduction d'Henriette Rouillard (1972) : Paris, Delagrave. (1re éd., 1935.)

- Traduction de René Bour (1951) : Lausanne, La Guilde du Livre. (1 éd., Paris, Paul Duval, 1938.)

- Traduction d'André Bay (1975) : Verviers, Marabout. (1re éd., Paris, Stock, 1947 ; $1^{\text {re }}$ trad. d'A. Bay, Paris, Stock, 1941.)

- Traduction de Jacques Papy (1975) : Paris, Gallimard. (1re éd., Paris, J.-J. Pauvert, 1961.)

- Traduction d'Henri Parisot (1976) : Paris, Aubier-Flammarion. (1re éd., Paris, Flammarion, 1968.) 
La nationalité des personnages d'un livre se reconnait généralement aux noms qu'ils portent. Dans Alice, certains personnages sont désignés par leur prénom, d'autres par leur nom générique, soit simple nom d'animal, comme la Souris, le Dodo, le Griffon, etc., soit nom tiré d'une expression anglaise proverbiale ou imagée, comme le Lièvre de Mars, ou le Chapelier Fou, soit nom qui rappelle leur état, par exemple la Cuisinière, la Duchesse, le Roi et la Reine de Coeur.

Les noms propres que l'on rencontre dans l'ouvrage de Carroll sont donc des prénoms. Certains ont la même forme en français qu'en anglais et ont toujours été conservés. C'est le cas du prénom de l'héroïne, Alice, qui, fort heureusement, existe dans les deux langues. Les autres fillettes citées dans le livre portent des prénoms moins courants en français, ce qui a gêné certains des traducteurs. Ainsi Ada et Mabel (37), les deux enfants en qui Alice a peur d'être changée, deviennent respectivement Adrienne et Madeleine (HR : 20). Ailleurs, Mabel subsiste mais Ada est transformée en Édith (JP : 32). Le nom des trois sœurs qui vivaient au fonds d'un puits, Elsie, Lacie et Tillie (100), a aussi parfois été modifié, sans doute parce que ces prénoms, et surtout les deux derniers, risquaient de paraître bizarres dans un contexte français. Dans l'une des versions, ils sont donc remplacés par "Marie, Annie et Lucie " (HR : 77). Un autre traducteur conserve Elsie, mais nomme ses deux sœurs Julie et Titi (RB : 119). Le lecteur anglais non averti ne reconnaîtra d'ailleurs pas plus que son homologue français dans Elsie, Lacie et Tillie le nom des trois filles du doyen de Christchurch College à qui Lewis Carroll avait raconté les aventures d'Alice, au cours d'une excursion en barque, le 4 juillet 1861 : Elsie est formé des initiales L.C. prononcées à l'anglaise, de la sceur aînée, Lorina Charlotte ; Lacie est l'anagramme d'Alice et Tillie est le diminutif de Matilda, nom donné dans sa famille à la troisième sœur, Édith.

Le nom du domestique irlandais du Lapin Blanc est francisé dans deux traductions : de Pat (60), il devient Patrice (HB : 49 et RB : 67), mais ce prénom ne dénote en français aucune origine particulière. Le nom de la femme de chambre du même lapin, Mary Ann (55), est orthographié à la française, soit Marianne (HB : 42, RB : 59, HP : 123), soit Marie-Anne (MMF : 38, HR : 37, AB : 48, JP : 53). Remarquons au passage les connotations historiques et politiques qui se greffent sur le prénom Marianne qui, depuis le Second Empire, désigne en France la République.

Father William, l'un des deux personnages de la parodie du poème de Southey "The Old Man's Comforts and How He Gained Them " qu'Alice récite à la Chenille (60-71), prend le nom de Guillaume, équivalent français de William, dans cinq versions (HB : 64, MMF : 53, HR:50, RB : 81, AB : 61). Les deux autres traducteurs le nomment "Père William " (JP : 70, HP : 145), appellation qui trahit l'origine étrangère du bonhomme. De fait, dans la première traduction allemande d'Alice, l'auteur et la traductrice se sentirent obligés de changer Father William en "Vater Martin " pour ne pas offenser le Kaiser ${ }^{4}$. Lewis Carroll préférait en effet donner aux personnages des traductions des noms aux consonances courantes dans la langue d'arrivée. C'est ainsi que Bill le Lézard devint Wabbel en allemand et Jacques en français (HB : 54).

Quant au nom du Lapin lui-même, "W. RABBIT », inscrit sur la porte de sa maison, il donne lieu à trois traductions différentes. Si l'initiale W. représente le prénom du Lapin, c'est aussi la première lettre de l'adjectif white. HR a supposé, pour sa part, que le personnage s'appelait William et l'a donc dénommé "Guillaume Lapin » (37). Plu-

Ces textes sont désignés dans notre article par les initiales de leur auteur.

Pour le texte original, nous avons utilisé : Lewis Carroll (1960) : The Annotated Alice, New York, Bramhall House, introduction et notes de Martin Gardner.

4. Derek Hudson, Lewis Carroll (1976) : Londres, Constable, p. 130. (1re éd., 1954.) 
sieurs autres traducteurs ont décidé de lui donner le nom que deux de ses congénères portent dans des fables de La Fontaine, Jean ou Jeannot ${ }^{5}$. On trouve ainsi "JEAN LAPIN » (HB : 42) et « J. LAPIN" (MMF : 40, AB : 48, HP : 125). RB (60) et JP (54) optent pour une autre désignation : ils font de "Lapin» le prénom et de "Blanc» le nom du personnage et l'inscription de la porte devient alors " $L$. BLANC ". L'appellation de "J. Lapin " a l'avantage d'évoquer immédiatement l'image du personnage animal qui habite la maison, même si elle ne suggère pas la couleur de son pelage.

Un autre indice qui permet au lecteur d'apprendre la nationalité des personnages est évidemment la langue qu'ils parlent. Dans l'original de l'ouvrage qui nous occupe, l'auteur mentionne à plusieurs reprises l'origine d'Alice et d'un certain nombre de ses interlocuteurs. L'attention du lecteur se trouve attirée sur la langue maternelle d'Alice lorsque la fillette, surprise de son changement de taille après avoir mangé un gâteau, s'exclame : "Curiouser and curiouser!' (she was so much surprised, that for the moment she quite forgot how to speak good English). " (35) Alice commet ici une faute de grammaire que l'auteur souligne en expliquant que d'habitude la fillette parle une langue correcte. La plupart des traducteurs ont choisi de rendre " to speak good English" par "s'exprimer " ou " parler correctement ", ce qui correspond en effet à la réalité : d'étonnement, Alice oublie sa grammaire française comme anglaise. RB a trouvé logique de remplacer l'anglais par le français dans le texte et sous sa plume, la fillette était "si surprise qu'elle avait pour le moment tout à fait oublié le bon français " (35). Cette solution est moins acceptable qu'elle ne le paraît au premier abord : elle " naturalise " Alice ainsi que les compagnons de ses aventures, alors que le récit conserve une toile de fond entrelacée d'éléments perçus comme étrangers dont nous verrons des exemples plus bas. De leur côté, les deux traductrices qui destinaient leur version à des enfants omettent aussi bien la faute de grammaire que la remarque de l'auteur qui l'accompagne (MMF : 21, HR : 17). Elles évitent ainsi la difficulté, mais on peut aussi se demander si elles n'ont pas cru bon de s'ériger en censeurs en supprimant une expression grammaticalement incorrecte.

Dans ce même chapitre, Alice est surprise de ce que la Souris, tombée comme elle dans la mare formée par ses larmes, ne comprenne pas l'anglais : Rerhaps it dosn't understand English" (...), "I daresay it's a French mouse, come over with William the Conqueror. "(41) En traduction, les personnages, dont Alice, ne parlent évidemment plus anglais. La supposition que la Souris ne comprend pas cette langue paraîtra donc bizarre au lecteur qui n'aura pas été prévenu de la nationalité de l'héroïne. Les traducteurs qui conservent telle quelle l'allusion à l'anglais le font par souci d'authenticité, mais il aurait sans doute convenu de préciser, en particulier dans les éditions pour enfants, l'origine du livre, par exemple dans une brève introduction, ce que fait seulement le volume de MMF et encore dans une notice visiblement destinée aux parents. Le premier traducteur avait bien senti la difficulté et avait ainsi rendu la réflexion que se faisait Alice : "Peut-être ne comprend-elle pas cette langue » (HB : 25). L'adjectif démonstratif est toutefois un peu vague et une remarque comme «Peut-être ne comprend-elle pas ce que je dis » aurait sans doute été plus adroite. Deux des traducteurs transposent carrément et font dire à la fillette : "Peut-être ne comprend-elle pas le français " (RB : 44, HR : 23). Nous avons déjà indiqué l'inconvénient de cette solution.

Au chapitre suivant, c'est son manque de clarté que l'un des personnages reproche à un autre en lui demandant de s'exprimer en anglais. Alice vient d'avouer qu'après avoir écouté l'histoire la plus sèche que la Souris connaisse, elle est tout aussi mouillée

5. La Fontaine (1954) : "Fables ", OEuvres complètes, t. 1, Paris, Gallimard. « L'Aigle et l'Escarbot ", 1. II, VII, p. 57 et «Le Chat, la Belette et le Petit Lapin», 1. VII, XVI, p. 176 
qu'avant. Sur quoi le Dodo déclare : "I move that the meeting adjourn, for the immediate adoption of more energetic remedies."

"Speak English !" said the Eaglet. "I don't know the meaning of half those long words, and what's more, I don't believe you do either. "(47) La demande de l'Aiglon fait sourire le lecteur qui sait bien que la langue utilisée par le Dodo est l'anglais, mais un anglais pompeux qui est en fait le jargon des comités. Aucun traducteur n'a conservé l'allusion à la langue anglaise qui aurait été ridicule ici. Les traductions se partagent en deux groupes. Deux font dire à l'Aiglon "Parlez français» (HB : 32, RB : 52), tandis que les autres lui font demander de parler "plus clair » (MMF : 33), "plus clairement " (AB : 39, HR : 29, HP : 113) ou "plus simplement " (JP : 43). Pourtant, aucune de ces solutions ne nous semble véritablement satisfaisante. En effet, il n'est pas souhaitable de transformer la nationalité des personnages puisqu'ils appartiennent visiblement à une culture étrangère. Quant au deuxième groupe des traductions, il a l'inconvénient de supprimer l'humour des paroles de Dodo. Nous aurions préféré une expression du genre de "Parlez comme tout le monde », tout en notant que le ton humoristique y aurait aussi été atténué.

Les allusions à la langue que parlent les personnages ont donc dû être transformées. Le rappel de la nationalité d'Alice et de ses compagnons est impossible par l'intermédiaire de la langue. Il est malgré tout souhaitable que le lecteur sache de quel pays ils sont originaires pour qu'il puisse s'expliquer un certain nombre de différences culturelles que nous allons examiner. D'où encore une fois l'importance d'une courte notice préliminaire indiquant la nationalité des personnages.

Le livre de Carroll contient des allusions à l'histoire d'Angleterre, mal connue des lecteurs français. Les deux références historiques que l'on y trouve se rapportent aux circonstances de l'accession au trône d'Angleterre de Guillaume le Conquérant, duc de Normandie. La bataille de Hastings est l'une des grandes dates de l'histoire d'Angleterre qu'apprennent tous les écoliers et comparable en importance dans l'histoire de France à la bataille de Poitiers ou celle de Bouvines. Mais Guillaume, envahisseur devenu roi, n'a pas d'équivalent exact dans les annales de la France. La plupart des traduc-. teurs ont d'ailleurs maintenu les allusions au duc de Normandie qui n'ont pas la même valeur en français qu'en anglais.

Nous avons déjà vu que, selon Alice, la Souris tombée dans la mare de larmes ne comprend pas l'anglais parce qu'elle est sans doute arrivée en Angleterre avec Guillaume le Conquérant. HB, peut-être poussé par l'auteur lui-même qui devait se rendre compte que le personnage cité n'était guère connu des petits Français, a supprimé l'allusion historique : "C'est sans doute une souris étrangère nouvellement débarquée " (25). Malheureusement, le vague du qualificatif " étrangère " et la disparition de la référence à un personnage historique bien connu affaiblissent singulièrement l'humour contenu dans la réflexion d'Alice. Deux traducteurs ont transposé en substituant à la conquête d'Angleterre par les Normands ... la Guerre de Cent Ans (RB : 44, HR : 23). RB ajoute même que c'est probablement en compagnie du Prince Noir que la Souris a dû traverser la Manche. Le renversement des nationalités paraît d'abord ingénieux mais il entraîne des complications. Dans l'original, l'hypothèse de la fillette sur l'origine de la Souris se trouve en quelque sorte confirmée par le récit que fait le rongeur au chapitre suivant de la façon dont Guillaume se vit offrir la couronne par les barons anglais.

William the Conqueror, whose cause was favoured by the pope, was soon submitted to by the English, who wanted leaders, and had been of late much accustomed to usurpation and conquest. Edwin and Morcar, the earls of Mercia and Northumbria, declared for him, and even Stigand, the patriotic archbishop of Canterbury, found it advisable... (47) 
Cet épisode de l'histoire de l'Angleterre est connu de tous les enfants anglais qui savent aussi que l'archevêque de Cantorbéry est le prélat le plus important de l'église anglicane. Par contre, les jeunes lecteurs français ignorent ces faits. Toutes les traductions conservent tel quel ce passage qui paraîtra encore plus " sec" en français. Dans les deux traductions qui ont transformé la conquête de l'Angleterre par les Normands en Guerre de Cent Ans, le lien entre l'origine de la Souris et l'histoire qu'elle raconte disparaît.

Alice renferme d'autre part des allusions géographiques qui reflètent soit les connaissances acquises par l'héroïne en classe, soit certains aspects de paysages anglais qu'elle a pu observer. Dans les deux cas, elles se rattachent pour elle comme pour ses compatriotes à une réalité familière, alors que le lecteur étranger les percevra différemment.

Ainsi la fillette se demande au cours de sa chute dans le terrier du Lapin si elle va traverser la terre de part en part et arriver chez les Antipathies qu'elle confond évidemment avec les antipodes. Elle sait que les pays situés aux antipodes de l'Angleterre sont la Nouvelle-Zélande et l'Australie, et donc elle envisage de demander à une passante : "Please, Ma'am, is this New Zealand or Australia?" (28) On s'explique mal pourquoi HB a traduit "New Zealand" par Nouvelle Zemble, nom d'un groupe d'îles russes de l'océan Glacial arctique qui ne se trouve certes pas aux antipodes de l'Europe occidentale. Quoi qu'il en soit, le nom des deux pays où Alice pense arriver n'a pas la même valeur en français et en anglais. Pour les sujets de la reine Victoria, les deux colonies britanniques de l'océan Pacifique faisaient partie de la réalité quotidienne : elles connurent toutes deux plusieurs vagues d'émigration après 1850 et il n'était pas rare d'avoir des parents ou amis établis dans ces contrées lointaines. Par contre, aucun lien ne rattache le lecteur français à ces pays où l'on ne parle pas sa langue.

L'auteur cite aussi dans Alice le nom de plusieurs comtés anglais, anciens comme la Mercie et la Northumbrie que nous avons déjà vus, ou moderne comme le Cheshire d'où est originaire le Chesthire Cat, animal grimaçant qui a la propriété d'apparaître et de disparaître à son gré (83). Les écoliers anglais apprennent la géographie de l'Angleterre anglo-saxonne et savent donc situer la Mercie et la Northumbrie. Le nom du comté de Cheshire leur sera aussi familier : même s'ils n'arrivent pas à le placer correctement sur une carte, ils y reconnaîtront un nom géographique semblable à ceux des autres comtés de leur connaissance, comme le Yorkshire, le Hampshire, etc. La plupart des traducteurs ont rendu le nom du Chat littéralement en l'appelant soit "Chat de Chester " (MMF : 65, HR : 61, RB : 96, AB : 75), soit "Chat du comté de Chester " (JP : 83), soit simplement "Chat du Cheshire" (HP : 163). Selon le Larousse, on emploie en effet pour désigner ce comté indifféremment la forme Chester (nom de son chef-lieu) et Cheshire. HB a pour sa part baptisé le félin de "Grimaçon " (84), appellation qui oblitère l'allusion géographique mais qui a l'avantage d'être suggestive puisqu'elle rappelle l'une des caractéristiques de l'animal qui est son sourire grimaçant.

Dans un ouvrage écrit à l'origine pour des enfants et qui mêle intimement fantaisie et réalité, il n'est pas surprenant de trouver des allusions aux méthodes d'enseignement utilisées à l'époque de sa composition. Les références à des manuels scolaires qu'employaient Alice, son frère et ses sœurs passent inaperçues de nos jours. Dans certains cas, cependant, il convenait d'effectuer une transposition. Ainsi, la question « Où est ma chatte? " (42) qu'Alice pose malencontreusement à la Souris était la première phrase de son livre de français ${ }^{6}$. Comme elle est indispensable à la suite du récit, aucun des traducteurs n'a pu la remplacer par un équivalent tiré d'un livre de classe français pour l'ap-

6. Note de Roger Lancelyn Green dans Lewis Carroll (1971) : Alice's Adventures in Wonderland et Through the Looking-Glass and What Alice Found There, Londres, Oxford University Press, p. 255. 
prentissage de l'anglais et tous ont donc conservé le sens. Pourtant, ceux d'entre eux qui ont fait de la fillette une petite Française lui font poser la question en anglais : "Where is my cat ? " (RB : 44, HR : 23). Mais dans la version d'HB où Alice suppose qu'elle a affaire à une souris "étrangère ", elle s'adresse à elle en italien : "Dove è il mio gatto ? " (25) Il est vrai que la question fera frissonner de peur le rongeur dans toutes les langues, mais sans que le lecteur y reconnaisse une phrase d'un manuel scolaire.

On peut aussi reconnaître des allusions didactiques dans les questions que se pose Alice au cours de sa chute dans le terrier du Lapin "Do cats eat bats? ? et «Do bats eat cats ? " (28), ainsi que dans celle que lui pose le Chat du Cheshire sur le sort advenu au bébé que berçait la Duchesse, "Did you say 'pig' or 'fig'? " (90) Il est possible d'y voir une réminiscence d'une méthode d'enseignement de la lecture : la méthode dite "phonic ", encore largement utilisée dans les pays de langue anglaise, a en effet recours à des groupes de mots qui riment, comme ceux que renferment ces questions. La traduction idéale de ces passages devrait donc faire apparaître une référence à une méthode d'apprentissage à la lecture. Pour ce qui est du premier cas cat/bat, par une heureuse coïncidence, les deux mots français désignant les deux animaux, bien que possédant un nombre de syllabes différent, commencent par le même phonème /š/ et pourraient donc servir à l'apprentissage de ce son. Par bonheur aussi, l'équivalent français de bat est une sorte particulière de souris et la traduction "Les chats mangent-ils les chauves-souris ? " et la question inverse sont parfaitement acceptables et même, dans une certaine mesure, plus logiques en français qu'en anglais. Les traductions du deuxième passage où figurent les mots pig et fig sont nettement moins satisfaisantes. Deux des traducteurs seulement ont trouvé des couples de lexèmes qui diffêrent uniquement par leur phonème initial. Dans une version, le Chat demande à Alice "Avez-vous dit cochon ou pochon ? (HP : 173), mais le deuxième mot est un mot rare : selon le Petit Robert, il est d'usage technique ou régional et désigne "une grande louche ". L'autre transposition a le désavantage d'utiliser des mots de trois syllabes, également moins courants que ceux de l'original : "Avez-vous dit porcelet ou corcelet ?" (HR : 68)

Une autre allusion aux méthodes d'enseignement a trait aux problèmes d'arithmétique que devaient résoudre les enfants anglais du temps où l'argent se comptait chez eux en livres, shillings et pence. Au cours du procès du Valet de cœur accusé d'avoir volé les tartelettes confectionnées par la Reine de Cour, le Roi demande au Chapelier, appelé à témoigner, quand il a commencé à prendre son thé. Le Chapelier, le Lièvre de Mars et le Loir donnent des réponses contradictoires et

the jury eagerly wote down all three dates on their states, and then added them up, and reduced the answer to shillings and pence (146).

Tous les traducteurs, à l'exception d'HP qui conserve shillings et pence (257-259), transforment les unités de monnaie en francs et centimes. L'action des jurés, tout en restant amusante à cause de son automatisme, perd alors tout son sens, évident en anglais pour qui a dû à l'école additionner des prix et donner le résultat en shillings et pence. Mieux aurait valu, en français, faire allusion à des problèmes d'arithmétique comportant des unités à convertir. Dans le domaine monétaire, par exemple, les traducteurs auraient pu choisir "sous" et "centimes", un sou valant cinq centimes.

Si l'élève anglais était rompu aux exercices de conversion de prix en shillings et en pences, il récitait aussi ses leçons dans une attitude différente de celle de son homologue français : les mains croisées sur les genoux. C'est précisément ce que fait Alice quand, pour se prouver qu'elle est bien elle-même et non Mabel, elle décide de réciter "How doth the little busy bee" : "she crossed her hands on her lap, as if she were saying lessons. " (38) Seuls deux traducteurs (RB : 39, AB : 30) procèdent à une adaptation et substituent à l'attitude de l'écolier anglais celle que prend d'ordinaire le petit Français 
qui se croise les bras. En cela, ils ont parfaitement raison : ce sont surtout les allusions indirectes aux méthodes d'enseignement qu'il convenait d'adapter. Les allusions directes comme celles à des manuels scolaires, par exemple, sont suffisamment explicites. Par contre, les références à la méthode d'apprentissage de la lecture ou à des problèmes d'arithmétique doivent être modifiées pour prendre toute leur valeur en traduction.

Un autre domaine qui occupe une place importante dans la vie des enfants est celui de la nourriture. La littérature enfantine se fait souvent l'écho de ces préoccupations et nombreux sont aussi les Nursery Rhymes qui contiennent des allusions à des préparations culinaires ou à l'action de boire ou de manger. Lewis Carroll mentionne également un certain nombre de plats, d'aliments et de boissons, et l'intérêt porté par Alice, dès que le Loir commence à raconter l'histoire des trois sœurs qui habitaient au fond d'un puits, notre héroïne veut aussitôt savoir de quoi elles se nourrissaient :

'What did they live on?' said Alice who always took a great interest in questions of eating and drinking. (100)

Ce qui nous intéresse en matière de traduction, c'est que l'expérience gastronomique varie d'un pays à l'autre. Lewis Carroll s'en était lui-même rendu compte lors de son unique voyage sur le Continent, et son Russian Journal fourmille de remarques, souvent humoristiques, à propos des différences culinaires qui l'avaient frappé?. Il ne précisait pas l'heure à laquelle il avait pris ses repas, mais il avait dû remarquer qu'elle changeait selon le pays où il se trouvait.

L'heure des repas et même leur appellation peuvent donc poser des problèmes au traducteur. Ainsi, le dinner marquait pour Alice la fin des leçons de la matinée. Si elle s'entendait bien avec le Temps, lui disait le Chapelier, il lui suffirait d'un mot pour que ce soit le moment de manger : "and round goes the clock in a twinkling ! Half-past one, time for dinner ! " (98) En anglais moderne, le dinner est de plus en plus le repas du soir, mais l'appellation des repas varie selon la classe sociale et aussi selon l'âge des convives : "U-speakers eat lunch in the middle of the day (...) and dinner in the evening. (...) Non-U speakers (also U-children and U-dogs), on the other hand, have dinner in the middle of the day ${ }^{8}$ " Alice, qui était un $U$-child, c'est-à-dire appartenant à la upper class, la bonne société, prenait donc son dinner au milieu de la journée, mais le terme anglais a des connotations sociales que ne possèdent pas en français "déjeuner " ou " dîner ". Parmi les traducteurs, HB est le seul à avoir utilisé " dîner " (105), comme on dit d'ailleurs aussi au Canada pour le repas du milieu de la journée. D'un autre côté, trois des versions françaises ont avancé l'heure de ce repas : il a lieu à midi (MMF : 77, HR:74) ou à midi et demi (AB : 89). À noter que ce sont les traducteurs qui destinaient leur texte à des enfants qui ont modifé l'heure pour ne pas dépayser leurs jeunes lecteurs.

Dans Alice, les animaux familiers ont droit à leur repas en même temps que les enfants. Le chien du fermier fait le beau pour demander son déjeuner : «it'll sit up and beg for its dinner. " (43) Le " dîner » qu'on trouve chez HR (24) et JP (38) sera compris en France comme désignant le repas du soir. Seul HP est fidèle à l'original en parlant de «déjeuner » (107). Les autres versions restent dans le vague et proposent " pour avoir à manger" (HB : 27), "pour demander sa pâtée " (MMF : 27), ou "pour obtenir à manger " (RB : 26).

7. Lewis Carroll (1935) : The Russian Journal, éd. par J.F. McDermott, New York, E.P. Dutton.

8. Alan S.C. Ross (1962) : "U and Non-U : An Essay in sociological Linguistics ", dans The Importance of Language, éd. par Max Black, Englewood Cliffs, N.J., Prentice-Hall, p. 101 (1re éd., in Noblesse oblige, éd. by Nancy Mitford, Londres, Hamish Hamilton, 1956). 
Mais le repas le plus typiquement anglais est le tea qui n'a pas d'équivalent exact en français et qu'on trouve à plusieurs reprises dans le livre de Carroll. Il convient d'ailleurs de distinguer entre tea-party et tea, comme nous allons le voir. Le chapitre VII s'intitule " $A$ Mad Tea-Party", le mot party indiquant la présence d'invités à qui l'on sert du thé accompagné de tartines, sandwiches et gâteaux. En France, dès le XVIII siècle, il était de bon ton, dans certains milieux, de boire du thé à la collation du soir, et à l'époque de la parution d'Alice, la Comtesse de Ségur décrivait le thé que Sophie, jouant à la dame, servait à ses amies et à son cousin ${ }^{9}$. Les enfants, eux, n'ont droit qu'à leur goûter et n'accompagnent que rarement leur mère quand elle va prendre le thé chez une amie. La plupart des traducteurs ont considéré la tea-party comme une réunion d'adultes et ont choisi le mot "thé". Fait exception HR qui dans sa version pour enfants emploie "goûter" (70). L'heure du thé, pourtant, comme celle du déjeuner, varie selon les traductions. Dans l'original, le Chapelier explique à la fillette que depuis sa dispute avec le Temps, c'est toujours l'heure du thé : "It's always six o'clock now » et «it's always teatime » (99). (Une note de Martin Gardner nous apprend qu'à l'époque, le five o'clock tea n'était pas encore entré dans les mœurs en Angleterre.) Les traducteurs semblent avoir craint que leurs lecteurs français ne trouvent bizarre l'heure tardive à laquelle le Chapelier et ses amis prennent le thé. Dans deux versions, elle est donc avancée de soixante minutes (MMF : 78, HR : 76). Quant à AB (91), il omet de préciser le moment exact de la journée, ce qui est, à notre avis, regrettable : "l'heure du thé » ne permet pas au lecteur français d'imaginer la position des aiguilles sur l'horloge.

La tea-party est donc une collation servie à des invités. Par contre, pour les enfants et les animaux anglais, le tea est simplement le repas du soir. C'est pourquoi Alice se dit en songeant avec nostalgie à sa chatte Dinah : "I hope they'll remember her saucer of milk at tea-time. " (28) Les traductions qui conservent « à l'heure du thé » (HB : 6, AB : 20, JP 20) montrent nettement que l'histoire est d'origine étrangère, car aucun chat français ne reçoit son lait à l'heure du thé. Il est plus normal que Dinah boive son lait "au goûter ", comme dans le texte de RB (21).

Quant aux préparations culinaires, Lewis Carroll en mentionne un grand nombre dans son livre. A. Chapel a bien montré qu'elles appartiennent à une expérience gastronomique spécifiquement anglaise ${ }^{10}$. La marmelade d'oranges est la confiture traditionnelle du breakfast britannique ; en France, elle demeure plutôt exotique. Les toasts (31) n'ont ni l'aspect ni le goût du pain grillé français et diffèrent aussi des biscottes. L'impression gustative produite par le contenu du flacon trouvé par Alice sur la table de verre ne peut pas être la même en français et en anglais puisque les ingrédients du mélange ne sont pas identiques : " it had, in fact, a sort of mixed flavour of cherry-tart, custard, pineapple, roast turkey, toffee and hot buttered toast. " (31) Il est bien vrai, comme le note A. Chapel, que la tarte aux cerises française ressemble fort à celle que l'on mange en Angleterre, que la dinde est le mets du repas de Noël dans les deux pays, que la crème appelée custard est plus épaisse que la crème à la vanille française et que l'ananas est un fruit plus rare outre Manche qu'en France. Aucun des traducteurs n'a donné comme équivalent de custard (la cossetarde du Canada français) "crème anglaise " qui est le terme le plus courant pour désigner ce dessert en France : évidemment, cette crème ne peut se nommer anglaise qu'en dehors de l'Angleterre. Le "flan " d'AB (23) est plus compact, de même que la "crème renversée " de JP (24). Plusieurs versions se contentent de proposer " crème ", mot trop peu précis pour qu'on l'associe à un goût particu-

9. Comtesse de Ségur (1960) : les Malheurs de Sophie, Paris, Hachette, chap. XII, «Le thé ", pp. 78-83 (1re éd., 1864).

10. Alain Chapel, op. cit., pp. 17-19. 
lier (HB : 11, MMF : 17, RB : 29). Ajoutons qu'HB, peut-être avec l'assentiment de l'auteur, a transformé la " dinde rôtie " en "dinde truffée ", mets encore plus délicat, et le toffee, genre de caramel mou, en "nougat». Le goût du mélange s'en trouve modifié, mais la valeur des composants est à peu près équivalente.

Les comfits, amandes ou fruits secs enrobés de sucre, étaient une confiserie de l'époque victorienne que ne connaissent guère les enfants anglais aujourd'hui ni même leurs parents. Des traductions proposées, à savoir "dragées » (HB : 34, MMF : 35, AB : 40, JP : 46, HP:115), « fruits confits " (RB : 53) et « bonbons " (HR : 30), c'est encore le premier terme qui convient le mieux, malgré les connotations différentes qu'il suggère. Passons rapidement sur les merlans couverts de chapelure et qui se mordent la queue : comme le fait remarquer A. Chapel, ils ne figurent jamais ainsi à un menu français. "They have their tails in their mouths and they're all over with crumbs " (136) est traduit littéralement sauf chez MMF (110) où le merlan devient un rouget, dans une tentative de conservation du jeu de mots sur whiting (" merlan " et "blanc à chaussures").

Le mock-turtle soup est également un plat typiquement anglais : c'est un potage à la tête de veau (d'où l'illustration par Tenniel de la Mock-Turtle avec un corps de tortue et une tête de veau), plus économique que le véritable consommé de tortue, pratiquement inconnu en France. Les lecteurs français ignorent que le potage à la vraie tortue est un mets de luxe, réservé aux grandes occasions et servi, par exemple, aux banquets offerts par le lord-maire de Londres. L'expression " fausse-tortue " se trouve déjà chez HB (140) et a été reprise par HR (95) mais c'est MMF qui a eu l'idée d'utiliser " Tortue à la tête de veau " $(100)$ en s'inspirant de la gravure de Tenniel. Ni la "Simili-tortue" (JP : 126), ni la " Tortue fantaisie » (HP 223) n'évoquent quoi que ce soit de précis dans l'esprit d'un lecteur français.

Signalons aussi le pie que se partagent le hibou et la panthère du poème que récite Alice au Griffon et à la Tortue :

I passed by his garden, and marked, with one eye,

How the Owl and the Panther were sharing a pie:

The Panther took pie-crust and gravy and meat,

While the Owl had the dish as its share of the treat. (140)

Ce genre de tourte ne se mange pas en France où le pâté ne contient pas de sucre. C'est pourtant le terme employé par tous les traducteurs, ce qui en oblige un à supprimer la sauce (JP : 143) et un autre à la remplacer par des miettes (HP : 247).

Les tartes dérobées par le Valet de Cour étaient, selon le Loir, des treacle tarts (151). Le treacle, "mélasse ", appelé d'ailleurs molasses en Amérique du Nord, est un résidu de la cristallisation du sucre, d'un emploi très rare en France. Le mot " mélasse " a des connotations très différentes de l'anglais treacle, puisque c'est un terme technique de l'industrie sucrière, qui désigne aussi dans le langage familier un brouillard dense ou une boue épaisse, ou, plus généralement, une situation confuse. Le lecteur trouvera donc étranges ces " tartelettes à la mélasse " qui figurent dans toutes les versions françaises d'Alice sauf une (RB : 182) qui propose des "tartes au sirop ", pâtisserie également inexistante.

La traduction des termes désignant les repas et les aliments soulève donc assez souvent des problèmes soit parce qu'il n'existe pas d'équivalent exact d'une langue à l'autre, soit parce qu'ils ont une valeur différente qui provient d'habitudes alimentaires différentes dans les deux pays.

Les traducteurs ont aussi dû faire face à quelques rares allusions au système politique et judiciaire. Ainsi, le chapitre III du livre s'intitule " $\boldsymbol{A}$ Caucus Race and a Long Tale. " (45) En effet, le Dodo suggère aux animaux tombés dans la mare de larmes qu'ils se livrent à une caucus race pour se sécher. Le lecteur retiendra surtout le désordre de 
cette course dont les participants s'éparpillent dans toutes les directions sans aucun but, mais où ils sont tous gagnants. Pourquoi l'auteur a-t-il dénommé cette course bizarre caucus race? Déjà à l'époque, caucus désignait aux États-Unis le groupe des représentants d'un même parti politique. En Angleterre, le mot prit un sens péjoratif et fut employé par les membres d'un des deux partis politiques pour désigner ceux du parti adverse. Selon l'OED, il ne serait devenu courant qu'après 1878 , c'est-à-dire après la parution d'Alice (1865). Les critiques s'accordent en général pour exprimer l'opinion que la caucus race de Carroll attaque le manque de méthode et d'objectifs précis des comités politiques et universitaires. Cette allusion disparaît complètement dans la plupart des traductions françaises. Déjà le premier traducteur intitulait le Chapitre III " La Course cocasse " (29), la compétition en question étant en effet burlesque et le mot caucus l'ayant fait penser à l'objectif " cocasse ". Trois des versions examinées reprennent le terme caucus, incompréhensible pour le lecteur français qui n'est pas au courant du système politique américain (MMF : 31, $\mathrm{AB}: 37, \mathrm{JP}: 41$ ). L'une des autres traductions propose précisément "Course à l'américaine" (HR:27), ce qui semble tout à fait arbitraire si le lecteur ne connaît pas l'original. Un autre texte traduit l'expression par "Une course à la cau cau as'u " (RB : 49), de façon aussi inexplicable qu'obscure. La seule traduction qui ait une valeur satirique est celle d'HP, "Une course à la comitarde " (109).

Les deux derniers chapitres de l'ouvrage décrivent le procès du Valet de Cœur. Les différences entre les systèmes judiciaires anglais et français n'apparaissent pas en traduction. Les jurys existent dans les deux pays et comme il s'agit d'un tribunal de fantaisie, peu importe que l'acte d'accusation soit lu par un " héraut » (herald, 145) et non par un greffier. Par contre, les juges anglais ne ressemblent pas à leurs confrères français : ils portent la perruque de laine alors que les magistrats français sont coiffés d'une toque. Or c'est à sa perruque qu'Alice reconnaît le juge, ce que le lecteur français ne comprendra guère ("That's the judge (...) because of his great wig. " (144) Certains traducteurs ont donc préféré coiffer le Roi d'une toque, à la manière d'un juge français. RB ajoute même à la tenue du Roi-juge une robe : "Voilà le juge (...) à cause de sa toque ronde et de sa robe " (171). Mais le texte de MMF où il est bien question d'une toque est orné d'une illustration où le Roi porte malgré tout la perruque des juges anglais. L'illustrateur n'avait sans doute pas eu accès à la traduction avant de produire ses gravures. Dans ce cas, la confusion du lecteur sera probablement plus grande que si la transposition de la perruque en toque n'avait pas été effectuée.

Nous n'insisterons pas sur les transformations des unités du système impérial ou " avoirdupoids " en unités du système métrique, qui ne sont pas particulières à la traduction d'Alice. Ce qui a gêné davantage les auteurs des différentes versions françaises du livre, ce sont les expressions imagées et les proverbes qu'il contient. Plusieurs personnages tirent leur nom d'expressions imagées ou proverbiales courantes en anglais britannique.

Ainsi, le Cheshire Cat dont nous avons déjà parlé, caractérisé aussi bien par son sourire que par son pouvoir d'apparaître et de disparaître à son gré, doit son existence à l'expression " to grin like a Cheshire Cat ", dont l'origine a donné lieu à des controverses. Le sourire grimaçant du Cheshire Cat est donc proverbial en anglais alors qu'en français, il devient absolument arbitraire. L'appellation suggestive de "Grimaçon ", proposée par HB (84), a l'inconvénient de ne pas indiquer qu'il s'agit d'un félin et élimine également le sourire au profit d'une pure grimace. Du moins est-elle plus " parlante" pour le lecteur français que l'appellation du "Chat du Cheshire" ou "de Chester".

C'est le Chat qui apprend justement à Alice l'existence des deux autres personnages qui doivent leur nom à des expressions proverbiales. La fillette lui a demandé quelle 
sorte de gens vivent dans les parages. " 'In that direction', the Cat said [...] ' lives a Hatter : and in that direction [...] lives a March Hare. Visit either you like : they're both mad.' " (89) En anglais, l'origine du nom de ces deux personnages est évidente : il vient des expressions mad as a hatter et mad as a March hare. Les chapeliers, en effet, étaient souvent atteints autrefois d'un empoisonnement au mercure, produit utilisé dans la fabrication du feutre, et souffraient de tremblements des membres et même d'hallucinations. Quant au Lièvre de Mars, sa folie est due à ce que le début du printemps est pour lui la saison des amours. Or il n'existe pas en français d'expressions semblables. La seule locution suggérant le degré de folie est " fou à lier ", impossible à employer dans le contexte qui se présente aux traducteurs. Toutes les versions examinées ont donc conservé aux deux personnages des appellations calquées sur l'anglais : le Chapelier et le Lièvre de Mars. Deux des traducteurs fournissent une note explicative (MMF : 73, AB : 317). Seul HB (92) a supprimé le nom du mois qui n'évoque rien dans l'esprit du lecteur français. Pourtant, un peu plus loin, Alice décide de rendre visite au Lièvre plutôt qu'au Chapelier, pour la raison suivante : "I've seen hatters before', [...] ' the March Hare will be much the more interesting, and perhaps as this is May, it won't be raving mad - at least not as mad as it was in March.' " (90) Le lecteur français comprendra mal l'explication d'Alice. HB a par conséquent également fait disparaître le deuxième argument de la fillette. Par contre, dans les autres versions, le raisonnement d'Alice sera dépourvu de sens et son incohérence qui n'existe pas dans l'original s'apparentera à celle des propos du Chapelier et du Lièvre.

L'ouvrage renferme également un certain nombre de proverbes, soit cités textuellement, soit déformés par l'auteur dans un but humoristique et dont la traduction s'avérera malaisée. Souvent le lecteur ne reconnaîtra la maxime ou l'expression proverbiale que si le traducteur l'y aide par son adresse.

Tel est le cas lorsque le Roi de Cœur réprimande le Chat du Cheshire qui a refusé de lui baiser la main : "Don't be impertinent, [...] and don't look at me like that !" (114) et qu'Alice prend la défense du Chat : " $A$ cat may look at a king, [...] I've read that somewhere, but I don't remember where. "Il s'agit là en fait d'un proverbe anglais signifiant que la différence de rang n'est pas nécessairement un obstacle aux relations et dont l'équivalent français serait : "Un chien regarde bien un évêque ". La plupart des traductions se contentent de rendre littéralement le proverbe anglais et l'une d'entre elles (JP : 118) explique en note que c'est un dicton dont elle fournit l'équivalent français. L'une (RB : 138) met dans la bouche d'Alice le proverbe français dont les deux personnages, le chien et l'évêque, n'ont aucun rapport avec le contexte. Une seule accole avec adresse les deux dictons : " Pourquoi, dit Alice, un Chat ne regarderait-il pas un roi ? Un chien regarde bien un évêque ! " (HP : 211)

Mais il est dans Alice un personnage qui aime tout particulièrement citer des proverbes : la Duchesse qui, au chapitre IX, ponctue chacune de ses réponses à la fillette à l'aide d'une morale qui est en fait un dicton.

La première morale énoncée par la Duchesse et qui n'a d'ailleurs que très peu de rapport avec ce qu'Alice vient de dire ("The game's going on rather better now ") est " oh 'tis love, 'tis love that makes the world go round ! "(120), dicton courant en anglais et qui figure dans l'Oxford Dictionary of Quotations parmi les citations anonymes. Les traducteurs ont presque tous traduit cette formule littéralement, sauf HR qui l'a censurée en attribuant à love le sens de " brotherly love » : "Et la morale de ceci est que l'amitié fait tourner le monde» (93), ce qui déforme singulièrement le sens de la phrase.

Parmi les autres dictons anglais qu'emploie la Duchessee, un seul n'est pas déformé : Alice vient de l'avertir que le flamant qui lui sert de maillet au jeu de croquet pourrait lui donner un coup de bec : "Very true, [...] flamingœs and mustard both bite. 
And the moral of that is 'Birds of a feather flock together.'" Ce à quoi Alice réplique, avec une parfaite logique : "Only mustard isn't a bird. " (121) La "morale » de la Duchesse est l'un des proverbes les plus courants de la langue anglaise et correspond au français «Qui se ressemble s'assemble ", plus abstrait, cependant. Ici, la plupart des traducteurs ont utilisé le dicton français, mais alors, quand Alice rétorque "Seulement la moutarde n'est pas un oiseau " (HB : 136), sa remarque n'est pas en contradiction avec ce que vient de dire la Duchesse. Plusieurs versions ont tenté, avec plus ou moins de bonheur, de combiner les dictons français et anglais, mais nous préférons la morale imaginée par RB et qui pourrait passer pour un proverbe véritable : "Oiseaux de même plumage volent à même rivage " (146).

La plupart du temps, la Duchesse estropie les maximes qu'elle cite. Ainsi, elle énonce "Take care of the sense and the sounds will take care of themselves" (121) où le lecteur britannique reconnaîtra une altération de "Take care of the pence and the pounds will take care of themselves", dont l'équivalent français serait "Les petites économies font les bonnes maisons ". Le mot clé du dicton recréé par la Duchesse est sense. En effet, à son affirmation sentencieuse que "Oh, 'tis love, 'tis love that makes the world go round!", Alice a répliqué que "Somebody (la Duchesse elle-même au chapitre VI) said [...] that it's done by everybody minding their own business ! 》 Ce à quoi la Duchesse riposte "Ah well! It means much the same thing. " L'idée de signification est donc essentielle. C'est pourquoi la version qui propose "Un chien vaut mieux que deux gros rats » (HB : 136), par déformation d'"Un tiens vaut mieux que deux tu l'auras ", n'est pas très adroite : il est impossible de rattacher le proverbe à ce qui précède et les paroles de la Duchesse semblent encore plus décousues que dans l'original. Dans les autres versions, l'analogie avec un dicton disparaît précisément parce que leurs auteurs n'ont fait que suivre le conseil de la Duchesse, "Take care of the sense."

Mentionnons enfin une autre expression employée par la Duchesse qui vient de trouver à Alice l'air songeur. La fillette réplique qu'elle a bien le droit de penser, ce que conteste la Duchesse : "Just about as much right as pigs have to fly. " (123) Or l'expression anglaise "when the pigs begin to $f y$ " correspond au français " quand les poules auront des dents ", ce qu'aucun des traducteurs n'a vu.

Dans l'ensemble, on constate qu'un certain nombre de personnages qui, dans l'original, doivent leur nom à des expressions anglaises ont en français un caractère tout à fait arbitraire. Non seulement ils ne se rattachent à aucun aspect de la réalité connue du lecteur, mais les réflexions que se fait Alice à leur sujet paraissent vides de sens et accroissent encore l'étrangeté du récit.

De même, les proverbes ou expressions proverbiales cités dans le texte n'ont pas d'équivalent exact. Une partie du message se trouve ainsi perdue et les propos de la $\mathrm{Du}$ chesse semblent encore plus extravagants en français qu'en anglais.

Les enfants de langue anglaise sont, dès leur plus jeune âge, nourris de chansons et formulettes, les nursery rhymes dont ils connaissent les paroles par cour. Si bien que les deux ou trois premiers mots d'une de ces poésies traditionnelles évoquent immédiatement dans leur esprit ceux qui suivent normalement et que les personnages qui peuplent ces courts récits plus ou moins fantastiques font partie de la réalité quotidienne d'une enfance anglo-saxonne. Les enfants à qui on lit Alice saisiront donc d'emblée les allusions aux nursery rhymes, même s'ils les rencontrent sous forme de pastiches. Ainsi, 
tout enfant d'expression anglaise réagira avec indignation à son premier contact avec les vers de Carroll

Twinkle, twinkle, little bat !

How I wonder what you're at!

Up above the world you fly,

Like a tea-tray in the sky. (98-99)

parodie de la poésie de Jane Taylor, qui figure dans tous les recueils de nursery rhymes :

Twinkle, twinkle, little star,

How I wonder what you are!

Up above the world so high,

Like a diamond in the sky.

Puis le jeune lecteur trouvera amusantes ces nouvelles paroles sans queue ni tête qui évoquent cependant des éléments de son monde quotidien, du moins en GrandeBretagne. Nous avons déjà vu que la chauve-souris, bat, figure souvent dans les méthodes d'apprentissage de la lecture. Le plateau à thé, lui, fait partie des ustensiles que comprend tout ménage britannique. La chauve-souris, animal plutôt répugnant, remplace donc l'étoile de la poésie pastichée, et le plateau à thé, objet commun, le diamant. Le sublime et le précieux font par conséquent place au répulsif et au vulgaire. Si nous ajoutons la note de fantastique que comporte la vision d'un plateau à thé traversant les airs, nous avons l'effet total, combinaison du banal et de l'insolite, sur le lecteur anglais.

En français, non seulement les chauves-souris et les plateaux à thé n'appartiennent pas au monde familier, mais l'impression de surprise causée par le remplacement des paroles connues par d'autres, absurdes, disparaît : la substitution passe inaperçue et il ne reste plus qu'une chanson dépourvue de sens. L'absurdité d'un vers comme « Scintille, ô ma chauve-souris ! (JP : 98) est purement gratuite et ne produit absolument pas le même effet que le vers correspondant en anglais.

L'autre nursery rhyme que l'on trouve dans l'ouvrage est citée textuellement par Carroll. Elle donne naissance à deux personnages du récit :

The Queen of Hearts, she made some tarts,

All on a summer day :

The Knave of Hearts, he stole those tarts

And took them quite away. (146)

La Reine de Cœur fait d'ailleurs son apparition bien avant que le Valet ne soit jugé pour le vol dont il est accusé à la fin du livre. Jusqu'au procès, donc, la Reine de Cour et son époux ne sont que des personnages de jeu de cartes. L'épisode du procès du Valet de Cour les replace pour le lecteur de langue anglaise dans un contexte qui fait partie des traditions de la petite enfance. Le chef d'accusation est connu d'avance : le premier vers de la poésie entraîne automatiquement la suite et le Valet ne peut qu'être coupable, d'autant plus que son nom anglais knave a aussi le sens péjoratif de "frippon " ou "fourbe ". Tout ceci passera inaperçu en français. Les traducteurs n'ont pu que rendre plus ou moins littéralement la substance de la petite poésie anglaise qui ne se rattache à rien dans l'esprit de leurs lecteurs.

Nous ne mentionnerons pas ici les autres parodies de poèmes que renferme Alice, bien qu'ils aient aussi constitué un écueil pour les traducteurs : les lecteurs modernes d'expression anglaise ne les perçoivent plus comme des pastiches puisque les originaux ont sombré dans l'oubli. Seuls deux traducteurs ont tenté de composer des pastiches comme équivalent de "How does the little crocodile" (38), parodie de "How doth the little busy bee " d'Isaac Watts : HB dans une fade imitation de la fable de La Fontaine " Le 
Corbeau et le Renard " (20), et AB qui a, dans sa version de 1941, pastiché "Le Coche et la Mouche " (28-29), pour ensuite revenir à une traduction plus fidèle du texte de Lewis Carroll.

Les problèmes culturels auxquels se sont heurtés les traducteurs d'Alice in Wonderland sont donc nombreux. Ils sont certainement responsables en partie de l'impression d'étrangeté que ressent le lecteur français au contact du livre. Même les plus habiles des traducteurs ne sont pas parvenus à le mettre à la portée du public français et on ne peut pas véritablement leur en tenir rigueur. Leur rôle d'intermédiaires entre deux cultures était loin d'être aisé. Cependant, s'il est vrai que le but d'une traduction est de rendre une œuvre étrangère accessible aux individus qui ne connaissent pas la langue dans laquelle elle a été écrite, il importe de ne pas les dérouter outre mesure. Or, les traducteurs ont assez souvent, semble-t-il, fait preuve d'un manque de méthode et ont assimilé les éléments culturels un peu au hasard. Dans ce domaine, c'est là le principal reproche que nous leur ferons, bien qu'en fait nous ayons surtout voulu montrer à quel point Alice in Wonderland était une œuvre nationale, difficile à apprécier pour un public non britannique. 